\title{
PEGADA HÍDRICA COMO INDICADOR DE SUSTENTABILIDADE EM POLO DE GRÃOS NA AMAZÔNIA
}

Douglas Cavalcante Costa ${ }^{1}$, Lucieta Guerreiro Martorano², Marcelo Coelho Marques $^{3}$, Jamil Chaar El-Husny ${ }^{4}$, Adelaide Nacif ${ }^{5}$

1 Mestrando em Agroecologia e Desenvolvimento Rural; Universidade Federal de São Carlos (UFSCar), e-mail: eng.douglascavalcante@gmail.com.

2 Doutora em Agrometeorologia; Pesquisadora na Embrapa Amazônia Oriental

3 Engenheiro Ambiental; Universidade do Estado do Pará

4 Doutor em Ciências Agrárias; Pesquisador na Embrapa Amazônia Oriental

5 Mestre em Geografia; Coordenadora Regional de Defesa Civil (CORDEC)/ SUDAM

Recebido em: 08/04/2016 - Aprovado em: 30/05/2016 - Publicado em: 20/06/2016 DOI: 10.18677/Enciclopedia_Biosfera_2016_079

\begin{abstract}
objetivo do trabalho foi avaliar a pegada hídrica das principais cultivares de soja como indicador de eficiência de uso da água em cultivos de grãos no município de Paragominas. Foi realizado trabalho de campo para acompanhar experimentos com soja visando obtenção de dados durante a safra agrícola 2013/2014. Considerou-se nas estimativas de pegada hídrica cinza o valor de $22 \mathrm{~kg} \mathrm{ha}^{-1}$ de nitrogênio, com base na literatura nacional. As cultivares de soja BRS Candeia; BRS Sambaíba; BRS Tracajá; BRS Seridó RCH; BRS Babaçu foram consideradas nas avaliações da pegada hídrica de acordo com o ciclo médio, disponível na literatura. Considerou-se os padrões limites de lançamento de fertilizante nitrogenado em corpos hídricos, adotando-se valores conforme a legislação no 357/2005 do Conselho Nacional de Meio Ambiente (CONAMA). Os resultados apontaram que a maior pegada hídrica foi na BRS Babaçu correspondendo a $1.306 \mathrm{~m}^{3}$ ton $^{-1}$ e a menor foi na BRS Candeia com $1.015 \mathrm{~m}^{3}$ ton $^{-1}$. Conclui-se que a pegada hídrica verde é um excelente indicador de eficiência de uso da água pela cultivar de soja utilizada em cada ano safra. Também, a quantidade de fertilizantes nitrogenados e o rendimento das cultivares são fatores determinantes na contabilidade de uso eficiente de água em polos grãos na Amazônia.
\end{abstract}

RESUMO

PALAVRAS-CHAVE: fertilizantes nitrogenados, rendimento, soja.

\section{WATER FOOTPRINT AS AN INDICATOR OF SUSTAINABILITY IN THE AMAZON GRAIN POLO}

\begin{abstract}
The study's goal was to evaluate the water footprint of all major soybean cultivars as use efficiency indicator of water in grain crops in the municipality of Paragominas. Fieldwork was conducted to monitor experiments with soy aiming at obtaining data for the $2013 / 2014$ harvest. It was considered in the gray water footprint estimates the
\end{abstract}


value of $22 \mathrm{~kg} \mathrm{ha}^{-1}$ of nitrogen, based on national literature. The soybean cultivars BRS Candeia; BRS Sambaíba; BRS Tracajá; BRS Seridó RCH; BRS Babaçu were considered in the evaluations of the water footprint according to the average cycle, available in the literature. It was considered the discharge limits patterns of nitrogen fertilizer in water bodies, adopting values according to the law No. 357/2005 of the National Council for the Environment (CONAMA). The results showed that the highest water footprint was in BRS Babaçu corresponding to $1,306 \operatorname{ton}^{-1} \mathrm{~m}^{3}$ and the smallest was in BRS Candeia with $1,015 \mathrm{~m}^{3} \mathrm{ton}^{-1}$. It follows that the green water footprint is an excellent indicator of efficiency of use of water the soybean cultivar used in each harvest year. Also, the amount of nitrogenous fertilizers and the yield of the cultivars are determining factors in accounting efficient use of water in grain poles in the Amazon.

KEYWORDS: soybeans, nitrogen fertilizers, yield.

\section{INTRODUÇÃO}

As ações que consideram aspectos sociais, econômicos e ambientais, apoiadas em políticas públicas propiciam elementos para avaliar indicadores de desenvolvimento sustentável em uma região. O conhecimento da capacidade de suporte aos impactos ambientais negativos por atividades antrópicas torna-se indispensável em avaliações de uso conservacionista (DA SILVA et al. 2013). Indicadores como a pegada hídrica (PH), a Pegada ecológica (PE) e a Pegada de carbono (PC) possuem metodologias capazes de subsidiar análises dos impactos ambientais gerados pelos padrões de consumo do planeta, que vão além da capacidade de suporte do Planeta (GALLI et al. 2012).

As pegadas hídrica e ecológica apesar de considerarem conceitos e métodos distintos possuem aspectos convergentes quanto ao uso dos recursos naturais, pois estes devem suprir as necessidades humanas. A PE trata de contabilizar o uso do espaço (hectares), enquanto, a PH contabiliza o volume de água doce utilizado na cadeia produtiva. Assim, a PH é uma ferramenta de gestão e planejamento dos recursos hídricos, visto que, a água doce representa apenas $2,5 \%$ do volume total do planeta (GLEICK, 2000).

A metodologia proposta por HOEKSTRA et al. (2011), evidencia que a PH é um forte indicador de manutenção ecossistêmica que apresenta caráter multidimensional em volumes de consumo de água por fonte de uso, bem como contabiliza os volumes de poluição, pelo tipo de atividade. A pegada hídrica é subdividida em Pegada Hídrica Verde (PHverde), Pegada Hídrica Cinza (PHcinza) e Pegada Hídrica Azul (PHazul). Assim, a PHverde considera o total de água precipitada que não escoa ou não repõe a água subterrânea, mas é armazenada no solo e utilizada pelas plantas, nos processos evapotranspiratórios. A PHcinza contabiliza o volume de água necessário para depurar efluentes. $\mathrm{Na}$ agricultura os produtos fitossanitários utilizados devem ser avaliados de modo que a qualidade da água se mantenha próxima ao seu estado natural, mantida acima de padrões mínimos de qualidade da água aceitáveis. Já a PHazul é um indicador do uso da chamada água azul, ou seja, a água doce superficial ou subterrânea que é utilizada em cultivos irrigados (HOEKSTRA \& CHAPAGAIN, 2007). A PH quantifica o uso da água na cadeia produtiva, avaliando o processo de produção, desde as ações do produtor até o consumidor final. No contexto agrícola, na cadeia produtiva deve-se considerar na PHtotal, ou seja, os somatórios das pegadas azul, verde e cinza. 
No Brasil, segundo a Associação Brasileira de Saúde Coletiva (Abrasco), a cultura da soja ocupa o $1^{\circ}$ lugar na classificação quanto ao uso de produtos fitossanitários. No Pará, a agricultura principalmente em Paragominas tem como cultura principal a soja (Glycine max). No ano 2000 o polo de grãos totalizou uma área plantada de 1.640 hectares e em 2012 foram destinados 62.354 hectares ao cultivo de soja, contribuindo para a expansão da produção agrícola brasileira, que nesse período aumentou mais 70\% (MEKONNEN et al., 2014). Com o aumento da sojicultura na região é necessário avaliar possíveis impactos ambientais negativos que podem surgir, principalmente quanto ao uso de fertilizantes utilizados nessas lavouras (COSTA et al., 2014). Portanto, a metodologia da PH surge como um importante indicador e planejamento do uso eficiente da água na cadeia produtiva da soja.

Diante do exposto, objetivou-se estimar a pegada hídrica das principiais cultivares de soja utilizadas no município de Paragominas para avaliá-la como um indicador de eficiência de uso da água em cultivos de grãos no município.

\section{MATERIAL E MÉTODOS}

\section{Área de estudo}

O experimento de campo foi realizado na Fazenda Poderosa (Figura 1),

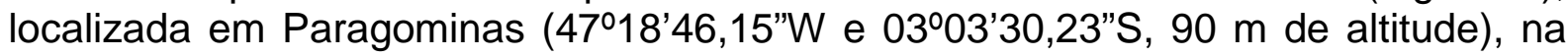
mesorregião sudeste do Estado do Pará, distante a $21 \mathrm{~km}$ da sede do município. Nessa propriedade tem sido realizado experimentos da Embrapa Amazônia Oriental, desde os anos 80 do século XX.

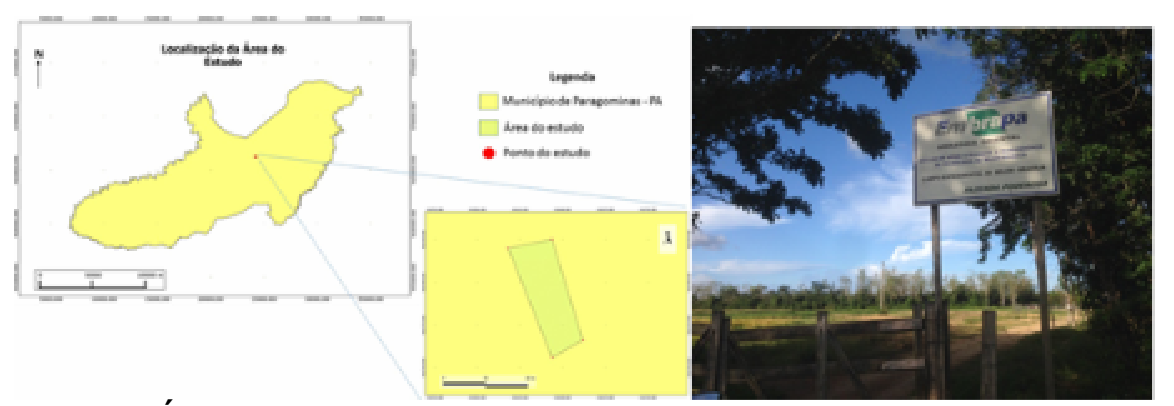

FIGURA 1 Área de estudo na fazenda Poderosa, Paragominas, Pará.

A fazenda contém uma área rural que totaliza 1.379,3 ha, composta de área destinada à reserva legal (695,6 ha) e de uso alternativo do solo (913,9 ha). Os experimentos incluem soja, milho, arroz, girassol até experimentos de longa duração com o de Castanha do Brasil.

\section{Dados das cultivares de soja}

A Tabela 1 apresenta os dados das cultivares, que foram utilizadas nas estimativas de $\mathrm{PH}$ verde e cinza em Paragominas, Pará. 
TABELA 1 - Cultivares, ciclo e rendimento de soja em Paragominas, com base em dados disponibilizados por EL-HUSNY et al. (2006)

\begin{tabular}{lcc}
\hline \multicolumn{1}{c}{ Cultivar } & Ciclo (dias) & Rendimento $\left(\mathbf{k g ~ h a}^{-1}\right)$ \\
\hline BRS Candeia & 118 & 3.975 \\
\hline BRS Sambaíba & 112 & 3.861 \\
\hline BRS Tracajá & 111 & 3.841 \\
\hline BRS Seridó RCH & 121 & 3.231 \\
\hline BRS Babaçu & 126 & 3.378 \\
\hline
\end{tabular}

\section{Levantamento de dados climáticos}

No ano de 2014 foram instalados instrumentos e sensores térmico-hídricos para avaliar quantidade de água precipitada e as condições térmicas e umidade relativa do ar na unidade experimental. Utilizou-se um pluviômetro Ville de Paris (030'08,4" S e 47ำ18'56,3" W) e sensor automático (Data loggers), inserido em mini-abrigo agrometeorológico, localizado nas coordenadas $03^{\circ} 03^{\prime} 08,4$ " $\mathrm{S}$ e $471^{\circ} 85^{\prime} 7,2$ " W. Os sensores foram instalados quando a soja estava em pleno desenvolvimento vegetativo (22 de abril de 2014) e retirado após o final do ciclo da (22 de junho de 2014) da cultivar que foi utilizada no experimento de campo (Figura 2). Destaca-se que coletas de eventos pluviais foram realizadas todos os dias por volta de 9:00 da manhã.

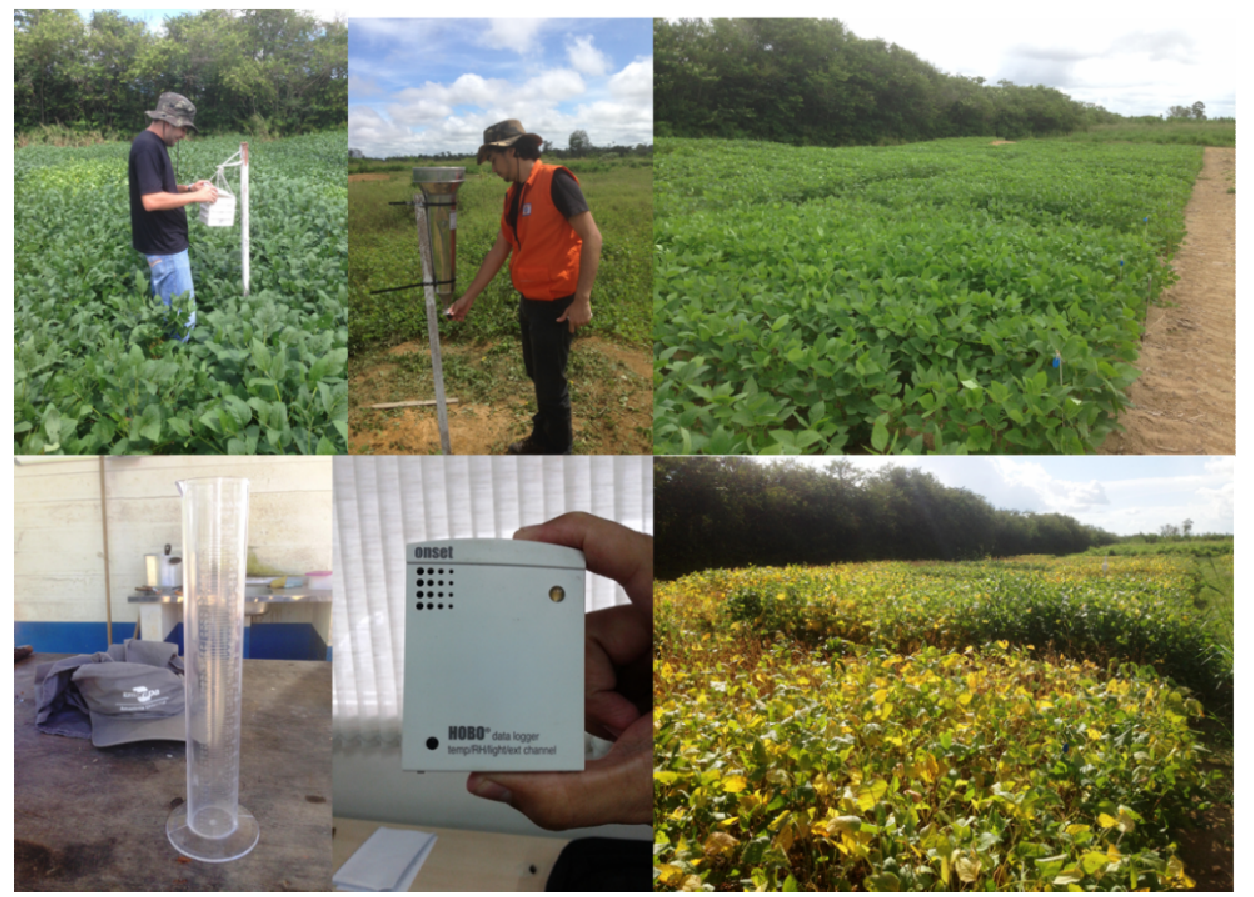

FIGURA 2: Equipamentos utilizados nas avaliações térmicohídricas em campo experimental

Para as estimativas da $\mathrm{PH}$, foram utilizados dados de precipitação pluvial do ano 2014, considerando-se os dados monitorados a campo, coletados seguindo os padrões recomendados pela Organização Meteorológica Mundial (OMM). Foram instalados mini-abrigos agrometeorológicos utilizando sensores térmicos. Também foram utilizados dados meteorológicos (temperatura, umidade relativa do ar, velocidade do vento e radiação solar), disponibilizados pelo Instituto Nacional de Meteorologia (INMET). 


\section{Pegada Hídrica Verde (PHverde)}

Para o cálculo de evapotranspiração da cultura, utilizou-se o software CROPWAT 8.0 que foi desenvolvido pela Organização das Nações Unidas para Agricultura e Alimentação (FAO, 2010). Os dados de coeficiente da cultura (Kc) foram os mesmos utilizados por ALLEN et al. (1998). A PH verde foi estimada baseando-se na chuva efetiva (Tabela 2) e as variáveis de cada cultivar apontadas na Tabela 1. Utilizou-se a metodologia de HOESKTRA et al. (2011), equação 1.

PHverde $=\frac{\text { DHCverde }}{\text { Prtv }}$

Em que:

$\mathrm{PHverde}=\mathrm{PH}$ verde $\left(\mathrm{m}^{3}\right.$ ton $\left.^{-1}\right) ; \mathrm{DHCverde}=$ demanda hídrica da cultura $\left(\mathrm{m}^{3} \mathrm{ha}^{-1}\right) ; \mathrm{e}$ prtv $=$ produtividade da cultura $\left(\right.$ ton ha $\left.{ }^{-1}\right)$.

O componente da DHCverde foi estimado com base na evapotranspiração diária acumulada $\left(\mathrm{ET}, \mathrm{mm} \mathrm{dia}^{-1}\right)$ durante o ciclo completo de desenvolvimento da cultura, conforme Equação 2 e 3.

ETverde $=\mathrm{ETo} \times \mathrm{Kc}$

DHCverde $=10 \times \sum_{\mathrm{d}=1}^{\mathrm{pdc}}$ ETverde

Em que:

ETverde = evapotranspiração da cultura, considerando apenas a oferta pluvial, sendo considerado por HOEKSTRA et al. (2011) como água verde.

$\mathrm{O}$ fator 10 refere-se a conversão da unidade de ET verde em milímetros para volumes de água por superfície terrestre, em $\mathrm{m}^{3} \mathrm{ha}^{-1}$. O somatório considera desde o dia de semeadura $(\mathrm{d}=1)$ até a colheita e pdc corresponde a duração do período de desenvolvimento da cultivar, adotada neste artigo o ciclo de cada cultivar.

TABELA 2 - Total de chuva e chuva efetiva no ano de 2014 em Paragominas, Pará.

\begin{tabular}{|c|c|c|}
\hline \multicolumn{3}{|c|}{2014} \\
\hline Meses & Chuva & Chuva Efetiva \\
\hline Janeiro & 280,8 & 200,6 \\
\hline Fevereiro & 309,4 & 223,5 \\
\hline Março & 256,1 & 180,9 \\
\hline Abril & 360,1 & 264,1 \\
\hline Maio & 251,5 & 177,2 \\
\hline Junho & 86,6 & 45,3 \\
\hline Julho & 32,7 & 9,6 \\
\hline Agosto & 18,2 & 0,9 \\
\hline Setembro & 16,4 & 0 \\
\hline Outubro & 28,1 & 6,9 \\
\hline Novembro & 40,4 & 14,2 \\
\hline Dezembro & 113,7 & 67 \\
\hline Total & 1794 & 1190,2 \\
\hline
\end{tabular}

ENCICLOPÉDIA BIOSFERA, Centro Científico Conhecer - Goiânia, v.13 n.23; p. 924 


\section{Pegada Hídrica Cinza (PHcinza)}

A estimativa da $\mathrm{PH}$ cinza foi realizada com base em fertilizante nitrogenado, empregado no processo de adubação de culturas, considerado como fonte difusa de poluição. Utilizou-se a metodologia de HOEKSTRA et al. (2011), Equação 4.

$\mathrm{PH}_{\text {cinza }}=\frac{(\alpha \times \mathrm{TAQ}) /\left(\mathrm{C}_{\text {máx }}-\mathrm{C}_{\text {nat }}\right)}{\operatorname{Prtv}}$

Em que:

$\alpha=$ fração de lixiviação do fertilizante.

$\mathrm{TAQ}=$ taxa de aplicação por hectare do composto em campo $\left(\mathrm{kg} \cdot \mathrm{ha}^{-1}\right)$.

Cmáx = concentração máxima aceitável.

Cnat = concentração natural do poluente no corpo hídrico.

\section{Parâmetros do fertilizante nitrogenado}

Neste trabalho atribuiu-se o valor de $10 \%$ para a fração de lixiviação, recomendado por HOEKSTRA et al. (2011). O valor limite aceitável dos fertilizantes nitrogenados seguiu o padrão da legislação CONAMA 357/2005, classe 3 para águas doces que é de $10 \mathrm{mg} \mathrm{L}^{-1}$. Atribuiu-se o valor 0 (zero) para a concentração natural do nitrogênio no corpo hídrico, pois nesse estudo não houve avaliações, análise e espacialização de corpos hídricos ao entorno das áreas agrícolas. $O$ dado de aplicação de nitrogênio para soja de alta produtividade foi a recomendada por LAMOND \& WESLEY (2001), que é de $22 \mathrm{~kg} \mathrm{ha}^{-1}$.

\section{RESULTADOS E DISCUSSÃO}

Com relação ao regime pluvial de Paragominas é possível observar na Figura 3 , em que os meses mais pluviosos concentram-se no período de janeiro a maio com chuvas variando entre 200 a $400 \mathrm{~mm}$, indicando a alta oferta hídrica pela atmosfera durante o ciclo dos cultivos de grãos no município.

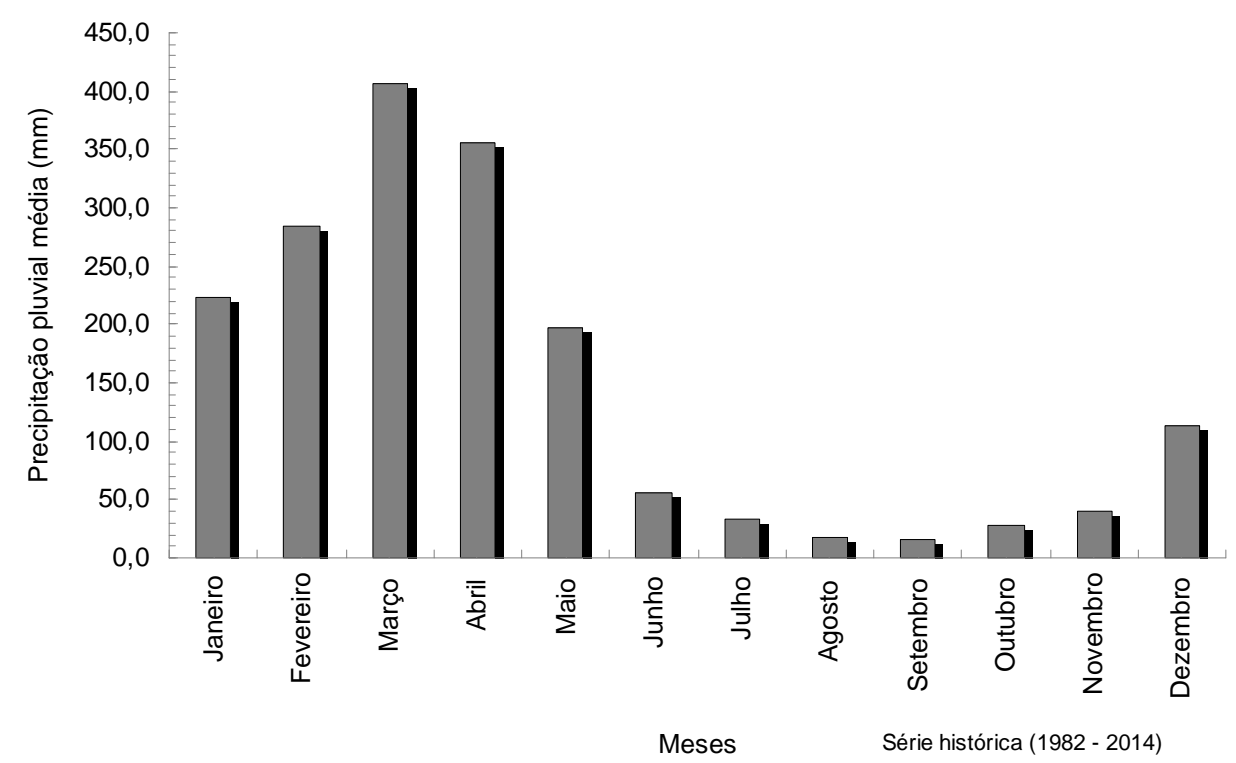

FIGURA 3: Precipitação pluvial média mensal no campo experimental, Paragominas, Pará. 


\section{Pegada hídrica}

Quando analisados os valores de $\mathrm{PH}$, observa-se que BRS Seridó $\mathrm{RCH}$ é a cultivar que mais necessita de água para o seu desenvolvimento $\left(1.327 \mathrm{~m}^{3} \mathrm{ha}^{-1}\right) \mathrm{e}$ com o menor rendimento de grãos $\left(3,2\right.$ ton $\left.\mathrm{ha}^{-1}\right)$, no ano safra avaliado por ELHUSNY et al. (2006). A BRS Candeia foi a cultivar que se destacou quanto a menor necessidade hídrica e maior rendimento de grãos, com $1.015 \mathrm{~m}^{3} \mathrm{ha}^{-1}$ e 4 ton ha ${ }^{-1}$, respectivamente.

Os valores de $\mathrm{PH}$ verde correspondem a cerca de $90 \%$ da $\mathrm{PH}$ total das cultivares, pois o sistema de produção da soja pouco utiliza fertilizantes artificias como o NPK, consequentemente terá valores de PH cinza reduzidos. Comparandose o resultado encontrado da $\mathrm{PH}$ da cultura da soja com os da literatura, têm-se: na região de Maringá com $2.210 \mathrm{~m}^{3}$ ton $^{-1}$ no estudo de BLENINGER \& KOTSUKA, (2015); $2.785 \mathrm{~m}^{3}$ ton $^{-1}$ por ERCIN \& HOEKSTRA (2012), 2.572,2 $\mathrm{m}^{3}$ ton $^{-1}$ por ARENA et al. (2011) , $1.385 \mathrm{~m}^{3}$ ton $^{-1}$ por HOEKSTRA \& MEKONNEN, (2011) e $1.958 \mathrm{~m}^{3}$ ton $^{-1}$ por HOEKSTRA \& CHAPAGAIN (2007).

Nota-se que os valores encontrados de $\mathrm{PH}$ no polo de grãos de Paragominas são condizentes com os da literatura. Ressalta-se que as diferenças de valores $\mathrm{PH}$ são justificadas pelas variáveis de solo, clima, manejo agrícola e genética da planta em cada área avaliada.

$\mathrm{Na}$ Figura 3 nota-se que os valores de $\mathrm{PH}$ cinza variam entre 55 a $67 \mathrm{~m}^{3}$ ton $^{-1}$, indicando a menor pegada cinza para a BRS Candeia. Em relação à Pegada Hídrica verde os valores variaram entre 960 a $1.239 \mathrm{~m}^{3}$ ton $^{-1}$, evidenciando as maiores eficiências de uso das águas pluviais em 2014 seriam também pela cultivar BRS Candeia, as quais refletiram nos valores de pegada hídrica total. Assim sendo, as diferentes cultivares testadas por EL-HUSNY et al. (2006) em Paragominas que também foram utilizadas no ano safra 2013/2014 deixam pegadas entre 1015 a $1.306 \mathrm{~m}^{3}$ ton $^{-1}$. Vale ressaltar que as estimativas de pegada verde se referem ao total de água utilizada pelas cultivares para completar seu ciclo em 2014.

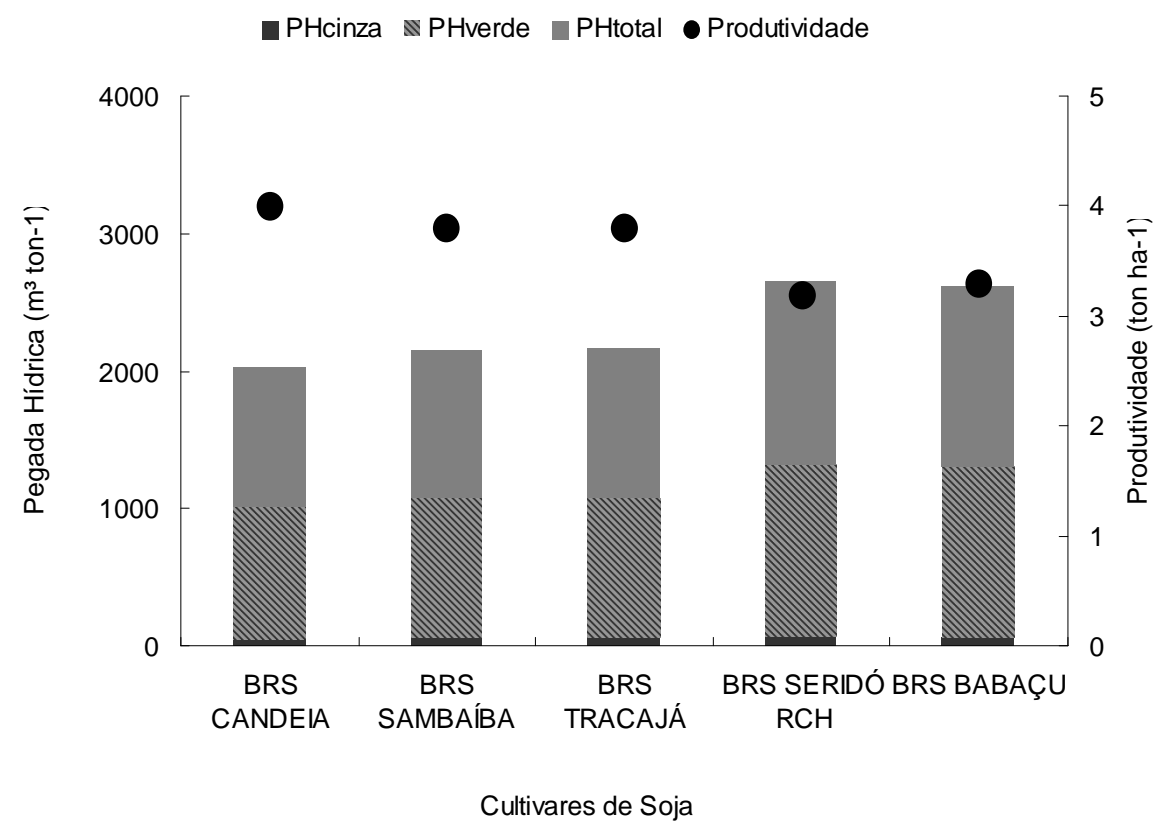

FIGURA 3: Estimativa da PH verde, cinza e total para as cultivares de soja. Fonte: Dados disponíveis em EL-HUSNY et al. (2006) 
Também, é importante salientar que o sistema de manejo do solo pode influenciar em cultivos não irrigados como, por exemplo em sistema com plantio direto que promove maiores estoques de água no solo em períodos com reduzida oferta pluvial, ao comparar com o sistema em preparo convencional (MARTORANO et al., 2009), indicando que outros fatores devem ser considerados nas avaliações de pegada hídrica.

Neste sentido, indicadores de sustentabilidade em sistemas de produção na Amazônia como a $\mathrm{PH}$ devem ser utilizados para as contabilidades de uso da água em sistemas de produção agrícola. A PH da soja fornece dados capazes subsidiar análises quanto aos avanços tecnológicos, sistema de manejo do solo e da água, como o plantio direto, integração Lavoura-Pecuária-Floresta (iLPF), uso de inoculantes em sementes de soja, doses de produtos fitossanitários e uso de tecnologia de alta eficiência de reposição hídrica. Assim, a pegada hídrica auxilia nas avaliações quanto ao uso eficiente da oferta de água na avaliação de indicadores de sustentabilidade no setor produtivo de grãos na Amazônia.

Algumas estratégias em sistemas de produção agrícola podem reduzir a $\mathrm{PH}$ da soja na Amazônia, tais como: otimizar épocas de semeadura em períodos com maior oferta hídrica nos polos de produção; adoção de sistemas de manejo do solo que garantam a manutenção da água para atender as demandas hídricas para expressar altos rendimento e baixa pegada hídrica da cultura; maior controle e fiscalização da entrada de novas moléculas de agroquímicos na Amazônia, com certificação da Agência Nacional de Vigilância Sanitária (ANVISA), em conformidade com as legislações do Conselho Nacional de Meio Ambiente (CONAMA); garantia de outorga de água para uso na agricultura; compartilhar conhecimentos regionais quanto a coeficientes genéticos, índices de colheita e testes de novas cultivares; e adoção de uma legislação específica voltada para o uso conservacionista da água capaz de garantir as demandas de consumo das gerações futuras na Amazônia.

\section{CONCLUSÃO}

- A pegada hídrica verde é um excelente indicador de eficiência de uso da água pela cultivar de soja utilizada em cada ano safra;

- A decisão na aplicação de fertilizantes nitrogenados influenciará na pegada hídrica cinza em sistemas produtivos de grãos na região;

- O rendimento de grãos em cultivos não irrigados na Amazônia é um ótimo aferidor nas avaliações de volume de água necessário para expressar as toneladas de soja colhidas em polos grãos;

- No ano safra 2013/2014 as menores pegadas hídricas verdes foram em cultivares de soja com rendimentos semelhantes as da BRS Candeia.

\section{AGRADECIMENTOS}

Os autores expressam seus agradecimentos à Embrapa Amazônia Oriental, principalmente ao Núcleo de Apoio a Pesquisa e Transferência de Tecnologia da Belém Brasília (NAPT Paragominas) pela autorização na condução do experimento de campo do trabalho de conclusão de curso (TCC) do primeiro e terceiro autor que originou esta publicação. 


\section{REFERÊNCIAS}

ALLEN, R. G.; PEREIRA, L. S.; RAES, D.; SMITH, M. 'Crop evapotranspiration: Guidelines for computing crop water requirements', FAO irrigation and Drainage Paper 56, Food and Agriculture Organization, Rome, 1998.

ARENA, A. P.; PIASTRELLINI, R; e CIVIT, B. Water Footprint of soybean production in Argentina. Life Cycle Management Conference, Berlim, Alemanha, 2011.

BLENINGER, T.; KOTSUKA, L. K. Conceitos de água virtual e Pegada Hídrica: Estudo de caso da soja e óleo de soja no Brasil. Revista Recursos Hídricos, v. 36, № 1, 15-24, 2015. Disponível em: <http://dx.doi.org/10.5894/rh36n1-2>. doi: $10.5894 / \mathrm{rh} 36 \mathrm{n} 1-2$

CONSELHO NACIONAL DO MEIO AMBIENTE - CONAMA. 2005. Resolução Conama no 357. Disponível em:< www.mma.conama.gov.br/conama> Acesso em: 08 abril 2014.

COSTA, D. C.; MARTORANO, L. G. ; MARQUES, M. C. ; LISBOA, L. S. ; BARBOSA, P. S. P. ; BARBOSA, A. M. S. . Estimativa da Pegada Hídrica Cinza no controle da Lagarta-da-soja (Anticarsia gemmatalis), no polo de produção de grãos Paragominas, Pará. In: Anais... XVIII Congresso Brasileiro de Meteorologia, Recife, 2014.

DA SILVA, V. P.R.; ALEIXO, D.O.; NETO, J.D.; MARACAJÁ, K.F.B.; DE ARAÚJO, L.E. Uma medida de sustentabilidade ambiental: Pegada Hídrica. Revista brasileira de Engenharia Agrícola e Ambiental, v.17, n. 1, p. 100-105, 2013. Disponível em: <http://dx.doi.org/10.1590/S1415-43662013000100014>. doi: 10.1590/S141543662013000100014

EL-HUSNY, J.C.; SILVEIRA FILHO, A.; ANDRADE, E.B.; CARVALHO, E.J; M.; BENCHIMOL, R. L.; VELOSO, C.A.C.V.; CORREA, J.R.V.; SOUZA, F.R.S.; Soja BRS Candeia: comportamento e recomendação para plantio nas microrregiões de Paragominas e Santarém. Embrapa Amazônia Oriental, Comunicado Técnico, 5p 182. Belém: Embrapa Amazônia Oriental, 2006.

ERCIN, A. E.; HOEKSTRA, A. Y.; Carbon and Water Footprints: Concepts, methodologies and policy responses. Unitec Nations World Water Assesnent Programme, Side Publications Series n.4, UNESCO, Paris. 2012.

FAO. 'CROPWAT $8.0 \quad$ Model'. FAO, Rome, www.fao.org/nr/water/infores_databases_cropwat.html, 2010

GALLI, A.; WIEDMANN, T.; ERCIN, E.; KNOBLAUCH, D.; EWING, B.; GILJUM, S. Integrating Ecological, Carbon and Water footprint into a "Footprint Family" of indicators: Definition and role in tracking human pressure on the planet. Ecological Indicators, v.16, p.100-112, 2012. Disponível em: <http://dx.doi.org/10.1016/j.ecolind.2011.06.017>. doi: 10.1016/j.ecolind.2011.06.017 
GLEICK, P. H. The changing water paradigm: A look attwenty-first century water resources development. Water International, v.25, p.127-138, 2000. Disponível em: <http://dx.doi.org/10.1080/02508060008686804. doi: 10.1080/02508060008686804

HOEKSTRA, A. Y.; CHAPAGAIN, A. K. Water Footprints of nations: water use by people as a function of their consumption pattern. Water Resources Management, v. 21, n. 1, p. 35-48, 2007. Disponível em: <http://dx.doi.org/10.1007/s11269-0069039-x>. doi: $10.1007 / \mathrm{s} 11269-006-9039-x$

HOEKSTRA, A. Y.; CHAPAGAIN, A. K.; ALDAYA, M. M.; MEKONNEN, M. M. Water Footprint Manual: Water Footprint Network. Enschede, the Netherlands.London: Earthscan; 2011.

HOEKSTRA, A. Y.; MEKONNEN, M. M. The water footprint of humanity. Department of Water Engineering and Management, University of Twente, P.O. Box 217, 7500 AE Enschede, The Netherlands. 2011. Disponível em: <http://dx.doi.org/10.1073/pnas.1109936109>. doi: 10.1073/pnas.1109936109

LAMOND, R.E.; WESLEY, T.L. Adubação nitrogenada no momento certo para soja de alta produtividade. Informações Agronômicas, n. 95, p. 6-7, 2001.

MARTORANO, L. G.; BERGAMASCHI, H.; DALMAGO, G. A.; FARIA R. T.; MIELNICZUK, J.; COMIRAN, F. Indicadores da condição hídrica do solo com soja em plantio direto e preparo convencional. Revista Brasileira de Engenharia Agrícola e Ambiental, v. 13, n. 4, p. 397-405, 2009. Disponível em: <http://dx.doi.org/10.1590/S1415-43662009000400005>. doi: 10.1590/S141543662009000400005.

MEKONNEN, M. M.; PAHLOW, M.; ALDAYA, M. M.; HOEKSTRA, A.Y. Water Footprint Assessment for Latin Amarica and the Caribbean: an analysis of the sustainability, efficiency and equitability of water consumption and pollution. Research Report Series No. 66. UNESCO-IHE, Delft, the Netherlands 2014. 